Dewey Ryan, M. (Orcid ID: 0000-0003-4437-0698)

Raines Jim, M (Orcid ID: 0000-0001-5956-9523)

Sun Wei-Jie (Orcid ID: 0000-0001-5260-658X)

Slavin James, A. (Orcid ID: 0000-0002-9206-724X)

Poh Gangkai (Orcid ID: 0000-0002-5775-2006)

\title{
MESSENGER observations of fast plasma flows in Mercury's magnetotail
}

\section{Ryan M. Dewey ${ }^{1}$, Jim M. Raines ${ }^{1}$, Weijie Sun ${ }^{1}$, James A. Slavin ${ }^{1}$, Gangkai Poh ${ }^{1}$}

${ }^{1}$ Department of Climate and Space Sciences and Engineering, University of Michigan, Ann Arbor, USA.

Corresponding author: Ryan M. Dewey (rmdewey@umich.edu)

\section{Key points:}

- Multiple FIPS plasma observations from the MESSENGER spacecraft have been combined statistically to determine average flows.

- Observations collected during dipolarizations produce an average plasma flow of $\sim 300$ $\mathrm{km} / \mathrm{s}$ compared to $\sim 50 \mathrm{~km} / \mathrm{s}$ during background intervals.

- Several dipolarizations are required to unload Mercury's magnetotail during a substorm, and some flows may reach the planet's surface.

Index terms: Mercury (6235); Magnetotail (2744); Substorms (2790); Instruments and techniques (2794)

Keywords: Mercury, magnetotail, bursty bulk flow, substorm, magnetic reconnection

Words: $\sim 3997$

Figures: 4

Tables: 0

Publication units: $~ 11.9$ (12 max)

This is the author manuscript accepted for publication and has undergone full peer review but has not been through the copyediting, typesetting, pagination and proofreading process, which may lead to differences between this version and the Version of Record. Please cite this article as doi: 10.1029/2018GL079056

This article is protected by copyright. All rights reserved. 


\begin{abstract}
We present the first observation of fast plasma flows in Mercury's magnetotail. Mercury experiences substorm activity phenomenologically similar to Earth's, however, field of view limitations of the Fast Imaging Plasma Spectrometer (FIPS) prevent the instrument from detecting fast flows in the plasma sheet. Although FIPS measures incomplete plasma distributions, subsonic flows impart an asymmetry on the partial plasma distribution, even if the flow directions are outside the field of view. We combine FIPS observations from 387 intervals containing magnetic field dipolarizations to mitigate these instrument limitations. By taking advantage of variations in spacecraft pointing during these intervals, we construct composite plasma distributions from which mean flows are determined. We find that dipolarizations at Mercury are embedded within fast sunward flows with an averaged speed of $\sim 300 \mathrm{~km} / \mathrm{s}$ compared to a typical background flow of $\sim 50 \mathrm{~km} / \mathrm{s}$.
\end{abstract}

\title{
Plain Language Summary
}

Similar to Earth, Mercury has a global magnetic field that forms a protective cavity, known as the magnetosphere, within the solar wind. The solar wind compresses the dayside magnetosphere, while stretching the nightside magnetosphere behind the planet. Variations within the solar wind cause dynamic activity within Mercury's magnetosphere, with a process known as magnetic reconnection mediating the interaction. Magnetic reconnection changes the topology of magnetic field lines and transfers energy and momentum from the magnetic field to the plasma within it. At Earth, magnetic reconnection in the nightside magnetosphere drives fast flows of plasma towards the planet, which when nearing the planet are slowed and diverted. These flows cannot be identified directly at Mercury because of limitations of the MESSENGER spacecraft measurements collected there. This research paper develops a new statistical technique to identify and characterize these fast flows at Mercury. 


\section{Introduction}

The MESSENGER spacecraft has observed that Mercury's magnetosphere experiences brief, yet intense, substorm activity characteristically similar to Earth's. Mercury's magnetotail exhibits loading/unloading (Slavin et al., 2010), dipolarization (Sundberg et al., 2012), plasmoid release (Slavin et al., 2009), energetic particle injection (Dewey et al., 2017), auroral-like precipitation (Lindsday et al., 2016), and current wedge formation (Poh et al., 2017). Mercury's substorms are significantly shorter and relatively stronger than Earth's, a result of the differences between the two magnetospheres (Siscoe et al., 1975). Mercury has a weak global magnetic field and lacks an ionosphere, but experiences stronger solar wind forcing that results in shorter temporal scales and higher magnetic reconnection rates than at Earth (e.g., Slavin et al., 2009; DiBraccio et al., 2013). The typical substorm cycle, for example, lasts $\sim 3 \mathrm{~min}$ at Mercury compared to the $\sim 1-3 \mathrm{~h}$ at Earth during which Mercury's lobe magnetic field strength increases on average by 23\% compared to the $\sim 10 \%$ at Earth (Imber \& Slavin, 2017; Forsyth et al., 2015; Hsu \& McPherron, 2000). While many features of Mercury's substorms have been identified and investigated, one major substorm signature has yet to be identified at Mercury - the presence of fast plasma flows in the magnetotail.

At Earth, bursty bulk flows (BBFs) are fast plasma flows within the plasma sheet, often traveling sunward with speeds $>400 \mathrm{~km} / \mathrm{s}$ (e.g., Angelopoulos et al., 1992) and typically accompanying magnetic field dipolarization (Ohtani et al., 2004). BBFs and dipolarizations follow the rapid reconfiguration of mid-tail region, $-30<X_{\mathrm{GSM}}<-15 \mathrm{R}_{\mathrm{E}}$ (where $\mathrm{R}_{\mathrm{E}} \sim 6371 \mathrm{~km}$ is Earth's radius), where x-lines drive explosive nightside energy release (e.g., Runov et al., 2012). The intense reconnection drives fast plasma flows that carry newly-reconnected dipolar field lines (dipolarizing flux bundle; e.g., Liu et al., 2013) toward the inner magnetosphere. As the dipolarizing flux bundle is carried planetward, the leading edge of the flux tube steepens to form 
the dipolarization front (e.g., Runov et al., 2009). Force balance (e.g., Karlsson et al., 2015) and specific entropy content (e.g., Wolf et al., 2009) determine the dynamics of the BBF as it moves planetward, resulting in rapid braking of the flow between $-15<X_{\mathrm{GSM}}<-10 \mathrm{R}_{\mathrm{E}}$ (e.g., Shiokawa et al., 1997; Fu et al., 2010) and in the generation of the substorm current wedge (e.g., Birn et al., 1999; Yao et al., 2012; Liu et al., 2013; Sun et al., 2013; Sergeev et al., 2014). During substorm intervals, BBFs contribute significantly to the mass, energy, and magnetic flux transport in the magnetotail (e.g., Angelopoulos et al., 1992; Liu et al., 2014; Schmid et al., 2016). Similar to Earth, fast plasma flows are expected in Mercury's magnetotail (e.g., Slavin et al., 2009; Sun et al., 2015a; Poh et al., 2017) in coincidence with dipolarizations (e.g., Sunberg et al., 2012; Dewey et al., 2017), which are a consistent signature of substorm activity at Mercury (e.g., Sun et al., 2015b).

Due to limitations imposed on the plasma instrument, MESSENGER cannot directly resolve plasma flows at Mercury. The spacecraft is 3-axis stabilized so the thermal ion sensor, the Fast Imaging Plasma Spectrometer (FIPS; Andrews et al., 2007), cannot measure complete plasma distributions from which to determine flows. Furthermore, the FIPS sensor never observes the sunward or antisunward directions since the spacecraft's sunshade must continuously point sunward. Although bulk flow cannot be determined unambiguously from an incomplete plasma distribution, a subsonic flow would impart asymmetry on the distribution even with the flow direction outside the field of view. In this study, we apply statistical techniques to identify flows in Mercury's magnetotail by combining multiple intervals to construct more complete plasma distributions. We find that similar to Earth, dipolarizations at Mercury are typically embedded in fast sunward flows. These are the first plasma flows measured at Mercury and illustrate the new capability of measuring statistical flows with FIPS.

\section{Data sources \& methodology}


FIPS measures thermal and low-energy ions with energy per charge ratio $(E / q)$ between $46 \mathrm{eV} / \mathrm{e}$ and $13 \mathrm{keV} / \mathrm{e}$ with a nominal energy scan time of $10 \mathrm{~s}$. FIPS is comprised of an electrostatic analyzer and a time-of-flight chamber, in the latter of which ions stop by encountering a position-sensing micro-channel plate (MCP). The stop MCP consists of an array of 64 by 64 pixels, each of which map to a location in the FIPS field of view (FOV), enabling the ions' incident direction to be determined. Combined, the MCP pixels allow for an instantaneous FOV imaging of $\sim 1.4 \pi \mathrm{sr}$ about FIPS's boresight direction (the central axis of the FOV cone), although spacecraft obstructions reduce this to an effective $\sim 1.15 \pi \mathrm{sr}$. We also use magnetic field vector measurements collected by the Magnetometer (Anderson et al., 2007) at $20 \mathrm{~Hz}$ resolution. We display all MESSENGER observations in the Mercury solar magnetospheric (MSM) coordinate system, which is centered at Mercury's dipole center with $X_{\text {MSM }}$ pointing sunward, $Z_{\text {MSM }}$ pointing northward, and $Y_{\text {MSM }}$ completing the right-handed system.

To identify fast flows, we analyze intervals containing dipolarizations selected by Dewey et al. (2017). Dewey et al. (2017) identified 538 dipolarizations coincident with energetic electron injections in Mercury's magnetotail from March 2013 to April 2015. An example of such an interval is shown in Figure 1a. During this 1-min interval, two dipolarizations are present: one beginning at $\sim 08: 26: 12$ and another at $\sim 08: 26: 40$. Both dipolarizations are coincident with enhancements in the Gamma-Ray Spectrometer (GRS) count rate, corresponding to energetic electron injections (e.g., Lawrence et al., 2015; Baker et al., 2016). The FIPS scan shaded in grey spans from the end of the first dipolarization to the beginning of the second. Figure $1 \mathrm{~b}$ contains the scan's angular flux map: proton flux accumulated during this scan as a function of MSM angular direction. Figure 1c contains the scan's angular FOV map: the number of MCP pixels observing each direction of MSM-space. Examining this scan's angular maps, the FIPS FOV limitations are apparent. The sensor surveys only a fraction of the sky and cannot observe plasma traveling in neither the sunward $\left(+X_{\mathrm{MSM}}\right)$ nor antisunward $\left(-X_{\mathrm{MSM}}\right)$ directions. While the sensor cannot unambiguously determine bulk plasma flow from the incomplete plasma distribution of 
this single scan, there is more flux traveling in $+X_{\mathrm{MSM}}$ than $-X_{\mathrm{MSM}}$ (see guiding arrows), suggestive of a sunward plasma flow. There are several high-flux bins near $-Z_{\mathrm{MSM}}$, however, these bins have high uncertainty as they are observed by few MCP pixels and correspond each to single proton counts.

Since a single FIPS scan has insufficient FOV coverage to determine plasma flows unambiguously, we construct composite plasma distributions by combining multiple FIPS measurements. Of the 538 Dewey et al. (2017) dipolarizations, we select 387 for statistical analysis. Figure 2 contains the equatorial distribution of all dipolarizations. For our analysis, we exclude regions near the magnetopause $\left(Y_{\mathrm{MSM}}<-1.5 \mathrm{R}_{\mathrm{M}}\right.$, where $\mathrm{R}_{\mathrm{M}} \sim 2440 \mathrm{~km}$ is Mercury's radius) to avoid contamination from the magnetosheath, regions close to the planet with poor viewing geometry (i.e., boresight pointing does not vary significantly across these events) to avoid biasing the composite distribution, and regions with too few events ( $<10$ dipolarizations) to avoid introducing outliers due to small geographic sample size. Of the 396 dipolarizations within the resulting region of interest (outlined by a thick black line), we exclude nine during which FIPS operated outside of its nominal mode.

To combine multiple FIPS scans into a composite plasma distribution, we: (1) construct a threedimensional spherical velocity phase space in MSM coordinates; (2) select all protons from the scans with corresponding MCP pixel location; (3) determine the velocity space location of each proton; (4) weigh each proton's phase space density (PSD) by the ratio of the solid angle of the MCP pixel that recorded it to the accumulated solid angle of all MCP pixels during that scan that observed that velocity space location; (5) add the weighted PSDs to velocity space; and (6) normalize the accumulated PSD at each velocity space location by the number of scans that observed that location. This procedure can be written as

$$
F(v, \theta, \phi)=\frac{1}{N(\theta, \phi)} \sum_{i=1}^{N(\theta, \phi)}\left[\sum_{j}\left(\Omega_{i j}(\theta, \phi) \sum_{k} f_{i j k}(v)\right) / \sum_{j} \Omega_{i j}(\theta, \phi)\right]
$$


where $(v, \theta, \phi)$ are typical spherical coordinates; $F(v, \theta, \phi)$ is the averaged PSD at velocity space location $(\nu, \theta, \phi) ; N(\theta, \phi)$ is the number of scans that observed $(\theta, \phi)$, indexed by $i$; $\Omega_{i j}(\theta, \phi)$ is the solid angle of MCP pixel number $j$ that observed $(\theta, \phi)$ during scan $i$; and $f_{i j k}(v)$ is the PSD of proton number $k$ that has velocity $(v)$ recorded by MCP pixel $j$ during scan $i$. Combining many FIPS scans with variable boresight pointing will generate a complete threedimensional plasma distribution except for the sunshade-blocked conic regions near $\pm X_{\mathrm{MSM}}$. Plasma flows along $\pm Y_{\text {MSM }}$ and $\pm Z_{\text {MSM }}$ can be determined unambiguously from this composite distribution while flows along $\pm X_{\text {MSM }}$ can be determined so long as they are sufficiently subsonic.

A composite plasma distribution from the 387 dipolarizations is shown in Figure 3. For this distribution, we combine all FIPS scans that occur in the $1 \mathrm{~s}$ before each dipolarization front midpoint. With a $1 \mathrm{~s}$ selection window, most dipolarizations contribute one FIPS scan to the composite distribution, however, $\sim 10 \%$ contribute two as one scan ends and another begins within the window, for a total of 424 scans. Clear anisotropies are observed in the angular flux map (Figure 3a). While $\pm X_{\text {MSM }}$ are not observed directly, there is greater flux traveling in $+X_{\text {MSM }}$ (yellow/orange) than $-X_{\mathrm{MSM}}$ (blue) surrounding the unobserved regions. There are bins with low flux (black) about both $+X_{\mathrm{MSM}}$ and $-X_{\mathrm{MSM}}$, however, these bins have high uncertainty as they are observed by few MCP pixels (Figure $3 b$ ). The $\pm X_{\text {MSM }}$ anisotropy is also observed away from the $X_{\mathrm{MSM}}-Y_{\mathrm{MSM}}$ plane. In the region between the $X_{\mathrm{MSM}}-Y_{\mathrm{MSM}}$ plane and $-Z_{\mathrm{MSM}}$, for example, greater flux is traveling in $+X_{\mathrm{MSM}}$ (green) than $-X_{\mathrm{MSM}}$ (blue). There is also clear anisotropy between $\pm Z_{\text {MSM }}$

Since the $\pm X_{\mathrm{MSM}}$ anisotropy is observed at all $Z_{\mathrm{MSM}}$ in the composite plasma distribution, sunward plasma flows are sufficiently subsonic to determine numerically. Calculating the moments (e.g., Paschmann et al., 1998) yields a proton density $n_{\mathrm{p}}$ of $0.60 \pm 0.03 \mathrm{~cm}^{-3}$, a sunward velocity $v_{\mathrm{x}}$ of $136 \pm 14 \mathrm{~km} / \mathrm{s}$, a duskward velocity $v_{\mathrm{y}}$ of $-60 \pm 25 \mathrm{~km} / \mathrm{s}$, a northward velocity $v_{\mathrm{z}}$ 
of $-140 \pm 18 \mathrm{~km} / \mathrm{s}$, and a proton temperature $T_{\mathrm{p}}$ of $38.6 \pm 0.9 \mathrm{MK}$. We evaluate uncertainties using both Monte Carlo and subsampling techniques. In the former, we perturb the PSD at each velocity space location by a random value from a normal distribution multiplied by that location's propagated Poisson error. In the latter, we randomly select $10 \%$ of the scans and construct a new composite plasma distribution. After generating new plasma distributions, we compute the new plasma moments. We repeat each technique with 5,000 iterations to form probability distributions for each plasma moment for each technique. For Monte Carlo, a distribution's spread represents that moment's instrument error, while for subsampling, it represents that moment's sampling error. We find that sampling error dominates instrument error.

We use a software model of the FIPS sensor (Dewey, Raines, and Tracy, 2017) to correct the plasma moments for the unobserved regions of the composite plasma distribution. The model simulates the sensor's response to a drifting Maxwellian plasma distribution. The model uses an input proton density, bulk velocity, temperature, and time-accurate pointing information to determine the PSD at each MCP pixel. Following the same procedure as for the composite plasma distribution, multiple intervals are combined and the plasma moments are calculated. Using this technique, we estimate that the in situ plasma in Figure 3 is most likely $n_{\mathrm{p}}=0.74 \pm$ $0.05 \mathrm{~cm}^{-3}, v_{\mathrm{x}}=225 \pm 25 \mathrm{~km} / \mathrm{s}, v_{\mathrm{y}}=-58 \pm 27 \mathrm{~km} / \mathrm{s}, v_{\mathrm{z}}=-147 \pm 18 \mathrm{~km} / \mathrm{s}$, and $T_{\mathrm{p}}=46.4 \pm 1.7$ MK. The moments determined directly from the composite plasma distribution underestimate $n_{\mathrm{p}}$ by only $\sim 20 \%, v_{\mathrm{x}}$ by $\sim 40 \%$, and $T_{\mathrm{p}}$ by $\sim 17 \%$ while capturing $v_{\mathrm{y}}$ and $v_{\mathrm{z}}$ well. At a temperature of $T_{\mathrm{p}}=46.4 \mathrm{MK}$, the thermal proton speed is $\sim 875 \mathrm{~km} / \mathrm{s}$, indicating the flow is subsonic with a Mach number of $\sim 0.3$.

\section{Results}

This article is protected by copyright. All rights reserved. 
To examine the evolution of the plasma flows about the 387 selected dipolarizations, we perform superposed epoch analysis on the plasma and magnetic field observations, displayed in Figure 4a. Each dipolarization is aligned at the midpoint of its dipolarization front (defined to be $t=0$ ). For each $1 \mathrm{~s}$ time step $-60<t<+40 \mathrm{~s}$, we collect the magnetic field, spacecraft location, and FIPS scans within the time window for each dipolarization. For each step, the average magnetic field and spacecraft location are calculated, and the plasma density, flow, and temperature are determined using the statistical technique described in Section 2. The composite plasma distribution from Figure 3 corresponds to the time of the dashed vertical line in Figure 4a.

Typical dipolarization signatures are immediately apparent. In $B_{\mathrm{z}}$, a decrease in the magnetic field beginning at $t=-3 \mathrm{~s}$ followed by a sharp, step-like increase to $t=+2 \mathrm{~s}$ marks the dipolarization front, while afterwards, the decaying $B_{\mathrm{z}}$ to a near-constant value $(+2<t<+7 \mathrm{~s})$ marks the dipolarizing flux bundle. The grey shaded region spans from the start of the dipolarization front to the end of the dipolarizing flux bundle. During this interval, the proton density decreases $\sim 30 \%$ and the proton temperature increases $\sim 20 \%$. The spacecraft is located, on average, in the post-midnight sector at local time $\sim 2.7 \mathrm{~h}$ and radial distance $\sim 1.5 \mathrm{R}_{\mathrm{M}}$, and its northward motion through the plasma sheet can be seen in the magnetic field components. $B_{\mathrm{x}}$ and $B_{\mathrm{y}}$ have small amplitudes throughout the interval but both reverse sign, consistent with the averaged spacecraft motion from $-Z_{\mathrm{MSM}}$ to $+Z_{\mathrm{MSM}}$, indicating a current sheet crossing. These composite plasma and magnetic field signatures are similar to previous studies at Mercury (Sundberg et al., 2012; Sun et al., 2017a), which is not surprising given the use of dipolarization intervals from Dewey et al. (2017).

A flow enhancement is observed coincident with the statistical dipolarization. Throughout the interval, $v_{\mathrm{x}}$ dominates the total flow speed $v_{\mathrm{t}}$, while $v_{\mathrm{y}}$ remains near $0 \mathrm{~km} / \mathrm{s}$ and $v_{\mathrm{z}}$ remains negative. Prior to the dipolarization $(-60<t<-30 \mathrm{~s})$, each velocity component remains steady with $v_{\mathrm{x}} \sim 160 \pm 22 \mathrm{~km} / \mathrm{s}, v_{\mathrm{y}} \sim 0 \pm 25 \mathrm{~km} / \mathrm{s}, v_{\mathrm{z}} \sim-100 \pm 20 \mathrm{~km} / \mathrm{s}$, and $v_{\mathrm{t}} \sim 200 \pm 18 \mathrm{~km} / \mathrm{s}$. From - 
$30<t<-15 \mathrm{~s}, v_{\mathrm{x}}$ increases steadily to $276 \pm 26 \mathrm{~km} / \mathrm{s}$ bringing $v_{\mathrm{t}}$ to $294 \pm 25 \mathrm{~km} / \mathrm{s}$. The flow speed remains at $\sim 300 \mathrm{~km} / \mathrm{s}$ until after the dipolarization $(t>+25 \mathrm{~s})$. During the dipolarization, the flow diverts azimuthally with $v_{\mathrm{t}}$ remaining constant while the magnitude of $v_{\mathrm{x}}$ decreases (228 $\pm 25 \mathrm{~km} / \mathrm{s})$ and the magnitude of $v_{\mathrm{y}}$ increases $(-74 \pm 27 \mathrm{~km} / \mathrm{s})$. The cyan arrow in Figure 2 marks the equatorial direction of the flow during the statistical dipolarization. Throughout the interval, $v_{z}$ remains negative, reaching a maximum absolute value of $-159 \pm 20 \mathrm{~km} / \mathrm{s}$ within the dipolarization at $t \sim+6 \mathrm{~s}$.

For comparison, we perform the same statistical analysis on quiescent intervals, shown in Figure 4b. We select 336 orbits between 1 March 2013 and 30 April 2015 that cross the magnetic equator within the region of interest (see Figure 2), contain no dipolarization-injection events (Dewey et al., 2017), and during which FIPS operated nominally. We align each orbit at the current sheet crossing $(t=0)$ and determine plasma and magnetic field parameters at $5 \mathrm{~s}$ resolution for times $-300<t<+300 \mathrm{~s}$. We select this time interval as it corresponds to the typical time required for the spacecraft to traverse $\left|Z_{M S M}\right|<0.5 R_{M}$, the region Dewey et al. (2017) used to identify dipolarizations. The shaded grey interval $(-20<t<+80 \mathrm{~s})$ corresponds to the same averaged $Z_{\mathrm{MSM}}$ traveled by the spacecraft as in Figure 4a. During this interval, plasma conditions remain constant to within uncertainty. The plasma sheet is denser $\left(n_{\mathrm{p}} \sim 3.10 \pm 0.26\right.$ $\left.\mathrm{cm}^{-3}\right)$, colder $\left(T_{\mathrm{p}} \sim 17.0 \pm 0.9 \mathrm{MK}\right)$, and more stagnant $\left(v_{\mathrm{x}} \sim 38 \pm 16 \mathrm{~km} / \mathrm{s}, v_{\mathrm{y}} \sim-7 \pm 23 \mathrm{~km} / \mathrm{s}, v_{\mathrm{z}}\right.$ $\sim 20 \pm 16 \mathrm{~km} / \mathrm{s}, v_{\mathrm{t}} \sim 47 \pm 17 \mathrm{~km} / \mathrm{s}$ ) compared to the averaged dipolarization and agrees well with previous typical plasma sheet proton densities and temperatures (e.g., Gershman et al., 2014). While the plasma moments show no significant trends within the shaded interval, the magnetic field is dominated by the spacecraft's motion through the planetary dipole field.

\section{Discussion \& Conclusions}


We use statistical techniques to determine mean proton flows in the plasma sheet during substorm and quiescent intervals using the FIPS data. We combine plasma observations from 387 dipolarization intervals and 336 background intervals to produce composite plasma distributions from which flows are inferred. During dipolarizations, the flow is $\sim 300 \mathrm{~km} / \mathrm{s}$ and predominately in the $+X_{\mathrm{MSM}}$ direction. By comparison, the convection speed during more quiescent intervals is $\sim 50 \mathrm{~km} / \mathrm{s}$. The dipolarization-associated flows are similar to those during dipolarizations at Earth. Liu et al. (2014), for example, found $v_{\mathrm{x}}$ to be typically $\sim 100 \mathrm{~km} / \mathrm{s}$ greater during a dipolarization than the interval preceding it.

While $v_{\mathrm{x}}$ is enhanced during dipolarizations, it increases in magnitude steadily prior to the dipolarizations, which may be associated with enhanced convection during the substorm growth phase. During the growth phase at Earth, enhanced convection driven by reconnection at the magnetopause pulls closed flux from the inner tail to the dayside reconnection region (Hsieh and Otto, 2014), which results in tail current sheet thinning (e.g., Sun et al., 2017b; Gordeev et al., 2017). Alternatively, this signature could be due protons reflected by the dipolarization front (e.g., Zhou et al, 2010) or an effect of averaging successive dipolarizations, as in Figure 1a. Nevertheless, an increase in $v_{\mathrm{x}}$ prior to a dipolarization is typically observed at Earth (e.g., Runov et al., 2011). Finally, the negative $v_{z}$ during dipolarizations may be combination of effects. It could be related to the spacecraft (located at $Z_{\mathrm{MSM}} \gtrsim 0$ ) observing current sheet thinning or the contraction of stretched field lines. It could also be related to the asymmetry between Mercury's loss cones, in which more particles are lost in the southern hemisphere, resulting in a net southward streaming and indicating particle loss. Sampling bias is unlikely to cause the $v_{\mathrm{z}}$ signature since the statistical composite technique accounts for FOV bias.

Given an average plasma sheet $B_{\mathrm{Z}} \sim 45 \mathrm{nT}$ and $v_{\mathrm{x}} \sim 250 \mathrm{~km} / \mathrm{s}$, the implied electric field during dipolarizations is $\sim 11 \pm 1 \mathrm{mV} / \mathrm{m}$. If we assume a single cross-tail flow channel width of $\sim 0.2 \mathrm{R}_{\mathrm{M}}$ (scaled from the $\sim 1-2 \mathrm{R}_{\mathrm{E}}$ at Earth), the additional cross-magnetospheric potential due to a 
dipolarization would be $\sim 5.4 \pm 0.5 \mathrm{kV}$ and the typical flux transported by a dipolarization would be $\sim 0.06 \pm 0.01 \mathrm{MWb}$. In contrast, from the statistical background observations, the typical cross-tail electric field is $\sim 2.4 \pm 1.2 \mathrm{mV} / \mathrm{m}$, corresponding to a cross-tail potential of $\sim 23 \mathrm{kV}$, which is consistent with previous estimates at Mercury (Slavin et al., 2010; DiBraccio et al., 2015; Jasinski et al., 2017). For substorm intervals, Imber \& Slavin (2017) found that loading typically increases the lobe magnetic content by $\sim 0.6 \mathrm{MWb}$ over a period of $\sim 100 \mathrm{~s}$. This rate of loading corresponds to a difference in the dayside and tail reconnection rates of $\sim 6 \mathrm{kV}$. While a single dipolarization can account for the reconnection rate difference, numerous dipolarizations $(\sim 10)$ are required to unload the magnetotail. Dipolarizations at Mercury, therefore, are associated with strong convection and transport although multiple are expected to occur during Mercury's substorm cycle.

Without reliable spatial gradients, the flow braking of the statistical dipolarization cannot be determined. As the dipolarization continues to move sunward, however, it is expected to encounter strong braking due to increased magnetic pressure gradients from the planetary dipole field (e.g., Shiokawa et al., 1997) where the flow's rapid braking and flux pile-up develop the substorm current wedge (e.g., Kepko et al., 2015). Using the magnetic field strength of the statistical dipolarization and a dipole description of Mercury's inner magnetotail (appropriate for radial distances $\lesssim 1.5 R_{\mathrm{M}}$; Rong et al., 2018), we estimate substantial braking to occur at radial distances $<1.3 R_{\mathrm{M}}$. If the negative $v_{\mathrm{z}}$ signature indicates particle loss as discussed above, the reduction in specific entropy of the dipolarizing flux tube would result in a braking region even closer to the planet (e.g., Wolf et al., 2008), with some fast flows possibly reaching Mercury's nightside surface. However, observations suggest that the typical dipolarization diverts about the planet (cyan arrow in Figure 2) such that it may not encounter steep gradients in the field and may instead propagate some distance before stopping. Without observations within the braking region, we cannot reliably estimate the typical dipolarization's contribution to the substorm current wedge, although the expectation that multiple dipolarizations are required to unload the 
magnetotail is similar to the wedgelet model at Earth (Liu et al., 2013). Interestingly, and requiring future investigation, a sustained series of dipolarizations compressing the nightside inner magnetosphere could produce induction effects in Mercury's core, similar to those induced on the dayside during strong solar wind forcing conditions (Slavin et al., 2014), which would cause the braking region to move tailward and possibly divert flows. Understanding the dipolarization flow speed as a function of downtail distance (e.g., Baumjohann et al., 1990) will help to refine these estimates; future observations from BepiColombo will be of particular value.

\section{Acknowledgements}

This research is supported by NASA's Discovery Data Analysis Program (NNX16AJ03G, NNX15AL01G), Living With a Star Program (NNX16AJ67G), Heliophysics Supporting Research Program (NNX15AJ68G), and Earth and Space Science Fellowship Program (80NSSC17K0493). All MESSENGER data used in this study are available from the NASA Planetary Data System.

\section{References}

Anderson, B. J., M. H. Acuña, D. A. Lohr, J. Scheifele, A. Raval, H. Korth, and J. A. Slavin (2007), The Magnetometer instrument on MESSENGER, Space Sci. Rev., 131, 417-450, doi:10.1007/s11214-007-9246-7.

Andrews, G. B., et al. (2007), The Energetic Particle and Plasma Spectrometer instrument on the MESSENGER spacecraft, Space Sci. Rev., 131, 523-556, doi:10.1007/s11214-007-92725.

Angelopoulos, V., W. Baumjohann, C. F. Kennel, F. V. Coroniti, M. G. Kivelson, R. Pellat, R. J. Walker, H. Lühr, and G. Paschmann (1992), Bursty bulk flows in the inner central plasma sheet, J. Geophys. Res., 97, 4027-4039, doi:10.1029/91JA02701.

Baker, D. N., et al. (2016), Intense energetic electron flux enhancements in Mercury's magnetosphere: An integrated view with high-resolution observations from MESSENGER, J. Geophys. Res. Space Physics, 121, 2171-2184, doi:10.1002/2015JA021778.

Birn, J., et al. (1999), Flow braking and the substorm current wedge, J. Geophys. Res. Space Physics, 104, 19,895-19,903, doi:10.1029/1999JA00173.

This article is protected by copyright. All rights reserved. 
Dewey, R. M., et al. (2017), Energetic electron acceleration and injection during dipolarization events in Mercury's magnetotail, J. Geophys. Res. Space Physics, 122, 12,170-12,188, doi:10.1002/2017JA024617.

Dewey, R. M., J. M. Raines, and P. J. Tracy (2017), Interpreting FIPS Density, Temperature, and Pressure, NASA Planetary Data System, MESS-E/V/H/SW-EPPS-3-FIPS-DDR-V2.0.

DiBraccio, G. A., et al. (2013), MESSENGER observations of magnetopause structure and dynamics at Mercury, J. Geophys. Res. Space Physics, 118, 997-1008, doi:10.1002/jgra.50123.

DiBraccio, G. A., et al. (2015), First observations of Mercury's plasma mantle by MESSENGER, Geophys. Res. Lett., 42, 9666-9675, doi:10.1002/ 2015GL065805.

Forsyth, C., et al. (2015), A new technique for determining substorm onsets and phases from indices of the electrojet (SOPHIE), J. Geophys. Res. Space Physics, 120, 10,592-10,606, doi:10.1002/2015JA021343.

Fu, H. S., et al. (2012), Occurrence rate of earthward-propagating dipolarization fronts, Geophys. Res. Lett., 369 L10101, doi:10.1029/2012GL051784.

Gershman, D. J., et al. (2014), Ion kinetic properties in Mercury's pre- midnight plasma sheet, Geophys. Res. Lett., 41, 5740-5747, doi:10.1002/ 2014GL060468.

Gordeev, E., V. Sergeev, V. Merkin, and M. Kuznetsova (2017), On the origin of plasma sheet reconfiguration during the substorm growth phase, Geophys. Res. Lett., 44, doi:10.1002/2017GL074539.

Hsieh, M.-S., and A. Otto (2014), The influence of magnetic flux depletion on the magnetotail and auroral morphology during the substorm growth phase, J. Geophys. Res. Space Physics, 119, 3430-3443, doi:10.1002/2013JA019459.

Hsu, T.-S., \& McPherron, R. L. (2000), The characteristics of storm-time substorms and nonstorm substorms, Proceedings of the International Conference on Substorms (ICS-5), Eur. Space Agency, Spec. Publ., ESA SP-443, 439.

Imber, S. M., \& Slavin, J. A. (2017), MESSENGER observations of magnetotail loading and unloading: Implications for substorms at Mercury, J. Geophys. Res. Space Physics, 122, 11,402-11,412, doi:10.1002/2017JA024332.

Jasinski, J. M., et al. (2017), Mercury's solar wind interaction as characterized by magnetospheric plasma mantle observations with MESSENGER. Journal of Geophysical Research: Space Physics, 122, 12,153-12,169, doi:10.1002/2017JA024594.

Karlsson, T., M. Hamrin, H. Nilsson, A. Kullen, and T. Pitkänen (2015), Magnetic forces associated with bursty bulk flows in Earth's magnetotail, Geophys. Res. Lett., 42, 31223128, doi:10.1002/2015GL063999.

Lawrence, D. J., et al. (2015), Comprehensive survey of energetic electron events in Mercury's magnetosphere with data from the MESSENGER Gamma-Ray and Neutron Spectrometer, J. Geophys. Res. Space Physics, 120, 2851-2876, doi:10.1002/2014JA020792. 
Lindsay, S. T., et al. (2016), MESSENGER X-ray observations of magnetosphere-surface interaction on the nightside of Mercury, Planet Space Sci., 125, 72-79, doi:10.1016/j.pss.2016.03.005.

Liu, J., V. Angelopoulos, A. Runov, and X.-Z. Zhou (2013), On the current sheets surrounding dipolarizing flux bundles in the magnetotail: The case for wedgelets, J. Geophys. Res. Space Physics, 118, 2000-2020, doi:10.1002/jgra.50092.

Liu, J., V. Angelopoulos, X.-Z. Zhou, and A. Runov (2014), Magnetic flux transport by dipolarizing flux bundles, J. Geophys. Res. Space Physics, 119, 909-926, doi:10.1002/2013JA019395.

Ohtani, S., M. A. Shay, and T. Mukai (2004), Temporal structure of the fast convective flow in the plasma sheet: Comparison between observations and two-fluid simulations, $J$. Geophys. Res., 109, A03210, doi:10.1029/2003JA010002.

Paschmann, G., A. N. Fazakerley, S. J. Schwartz (1998), Moments of plasma velocity distributions, Analysis Methods for Multi-Spacecraft Data, ISSI Scientific Report, SR001.

Poh, G., et al. (2017) Coupling between Mercury and its nightside magnetosphere: Cross-tail current sheet asymmetry and substorm current wedge formation, J. Geophys. Res. Space Physics, 122, 8419-8433, doi:10.1002/2017JA024266.

Rong, Z. J., et al. (2018), The magnetic field structure of Mercury's magnetotail, Journal of Geophysical Research: Space Physics, 123, 548-566, doi:10.1002/2017JA024923.

Runov, A., V. Angelopoulos, M. I. Sitnov, V. A. Sergeev, J. Bonnell, J. P. McFadden, D. Larson, K.-H. Glassmeier, and U. Auster (2009), THEMIS observations of an earthwardpropagating dipolarization front, Geophys. Res. Lett., 36, L14106, doi:10.1029/2009GL038980.

Runov, A., et al. (2011), A THEMIS multicase study of dipolarization fronts in the magnetotail plasma sheet, J. Geophys. Res., 116, A05216, doi:10.1029/2010JA016316.

Runov, A., V. Angelopoulos, and X.-Z. Zhou (2012), Multipoint observations of dipolarization front formation by magnetotail reconnection, J. Geophys. Res., 117, A05,230, doi:10.1029/2011JA017361.

Schmid, D., et al. (2016), A comparative study of dipolarization fronts at MMS and Cluster, Geophys. Res. Lett., 43, 6012-6019, doi:10.1002/2016GL069520.

Sergeev, V. A., et al. (2014), Stopping flow bursts and their role in the generation of the substorm current wedge, Geophys. Res. Lett., 41, 1106-1112, doi:10.1002/2014GL059309.

Shiokawa, K., W. Baumjohann, and G. Haerendel (1997), Braking of high-speed flows in the near-Earth tail, Geophys. Res. Lett., 24, 1179-1182, doi:10.1029/97GL01062.

Siscoe, G. L., Ness, N. F., \& Yeates, C. M. (1975), Substorms on Mercury? Journal of Geophysical Research, 80, 4359-4363, doi:10.1029/JA080i031p04359.

Slavin, J. A., et al. (2007), MESSENGER: Exploring Mercury's magnetosphere, Space Sci. Rev., 131, 133-160, doi:10.1007/s1/214-007-9154-x.

This article is protected by copyright. All rights reserved. 
Slavin, J. A., et al. (2009), MESSENGER observations of magnetic reconnection in Mercury's magnetosphere, Science, 324, 606-610, doi:10.1126/science.1172011.

Slavin, J. A., et al. (2010), MESSENGER observations of extreme loading and unloading of Mercury's magnetic tail, Science, 329, 665-668, doi:10.1126/science.1188067.

Slavin, J. A., et al. (2012), MESSENGER and Mariner 10 flyby observations of magnetotail structure and dynamics at Mercury, J. Geophys. Res., 117, A01215, doi:10.1029/2011JA016900.

Slavin, J. A., et al. (2014), MESSENGER observations of Mercury's dayside magnetosphere under extreme solar wind conditions, J. Geophys. Res. Space Physics, 119, 8087-8116, doi:10.1002/2014JA020319.

Sun, W. J., et al. (2013), Field-aligned currents associated with dipolarization fronts, Geophys. Res. Lett., 40, 4503-4508, doi:10.1002/grl.50902.

Sun, W.-J., et al. (2015a), MESSENGER observations of Alfvénic and compressional waves during Mercury's substorms, Geophys. Res. Lett., 42, 6189- 6198, doi:10.1002/2015GL065452.

Sun, W.-J., et al. (2015b), MESSENGER observations of magnetospheric substorm activity in Mercury's near magnetotail, Geophys. Res. Lett., 42, 3692-3699, doi:10.1002/2015GL064052.

Sun, W. J., et al. (2017), MESSENGER observations of the energization and heating of protons in the near-Mercury magnetotail, Geophys. Res. Lett., 44, 8149-8158, doi:10.1002/ 2017 GL074276.

Sun, W. J., et al. (2017b), Plasma sheet pressure variations in the near-Earth magnetotail during substorm growth phase: THEMIS observations, J. Geophys. Res. Space Physics, 122, doi:10.1002/2017JA024603.

Sundberg, T., et al. (2012), MESSENGER observations of dipolarization events in Mercury's magnetotail, J. Geophys. Res., 22, A00M03, doi:10.1029/2012JA017756.

Wolf, R. A., Y. Wan, X. Xing, J.-C. Zhang, and S. Sazykin (2009), Entropy and plasma sheet transport, J. Geophys. Res., 114, A00D05, doi:10.1029/2009JA014044.

Yao, Z. H., et al. (2012), Mechanism of substorm current wedge formation: THEMIS observations, Geophys. Res. Lett., 36, L13102, doi:10.1029/2012GL052055.

Zhou, X.-Z., V. Angelopoulos, V. A. Sergeev, and A. Runov (2010), Accelerated ions ahead of earthward propagating dipolarization fronts, J. Geophys. Res., 115, A00I03, doi:10.1029/2010JA015481.

This article is protected by copyright. All rights reserved. 


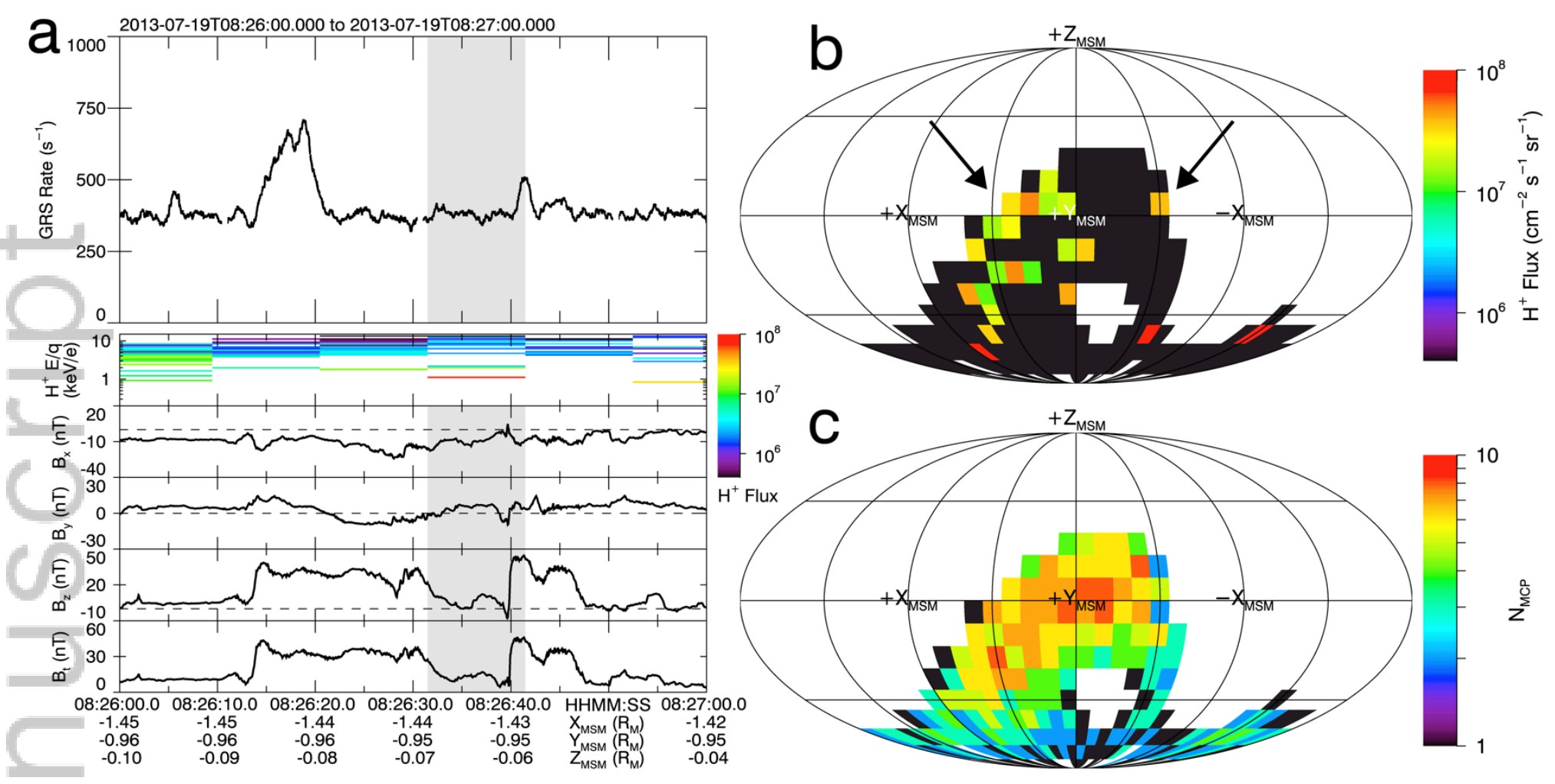

Figure 1. (a) Magnetic field dipolarizations and energetic electron injections identified by Dewey et al. (2017). From top to bottom: GRS count rate; FIPS $\mathrm{H}^{+}$flux spectrogram; and magnetic field components $B_{\mathrm{x}}, B_{\mathrm{y}}, B_{\mathrm{z}}, B_{\mathrm{t}}$. Spacecraft position is listed below the bottom panel. (b) FIPS angular flux map corresponding to the energy scan shaded in grey in (a). Color bins have nonzero flux as indicated by the upper color bar. (c) Angular FOV map of the same scan. The number of MCP pixels sampling each region of MSM space is indicated by the lower color bar. For both maps, white indicates regions outside the FOV. 


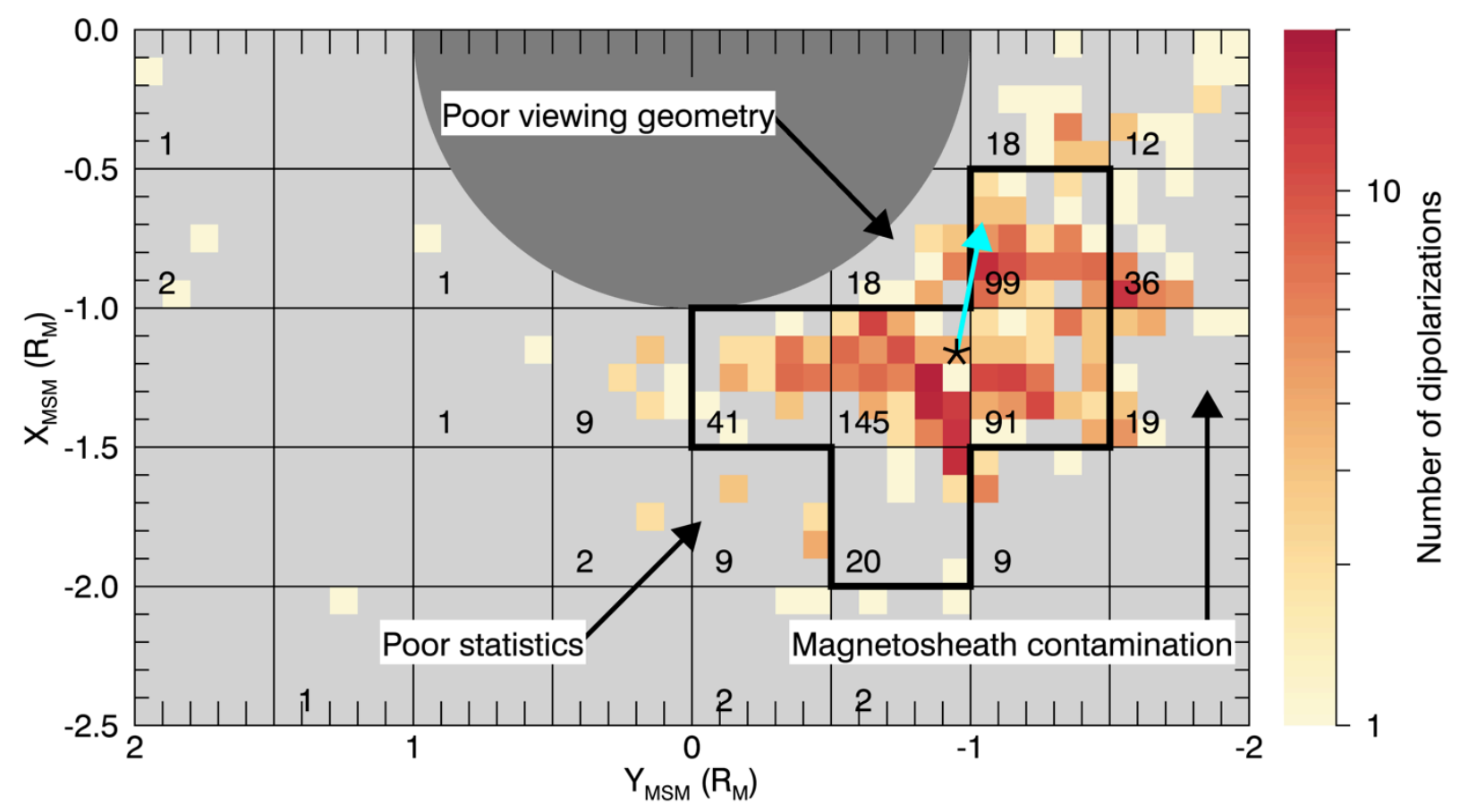

Figure 2. Equatorial distribution of dipolarizations identified by Dewey et al. (2017). The color bar indicates the number of dipolarizations within each $\left(0.1 \mathrm{R}_{\mathrm{M}}\right)^{2}$ bin; light grey corresponds to no dipolarizations. The number of dipolarizations within each $\left(0.5 \mathrm{R}_{\mathrm{M}}\right)^{2}$ box is listed in the box's lower-left corner. The thick black line outlines the region used for statistical analysis. For the selected dipolarizations, the star denotes the average spacecraft location and the cyan arrow points in the statistical equatorial flow direction. The dark grey region marks Mercury's surface. Annotations are discussed in the text. 


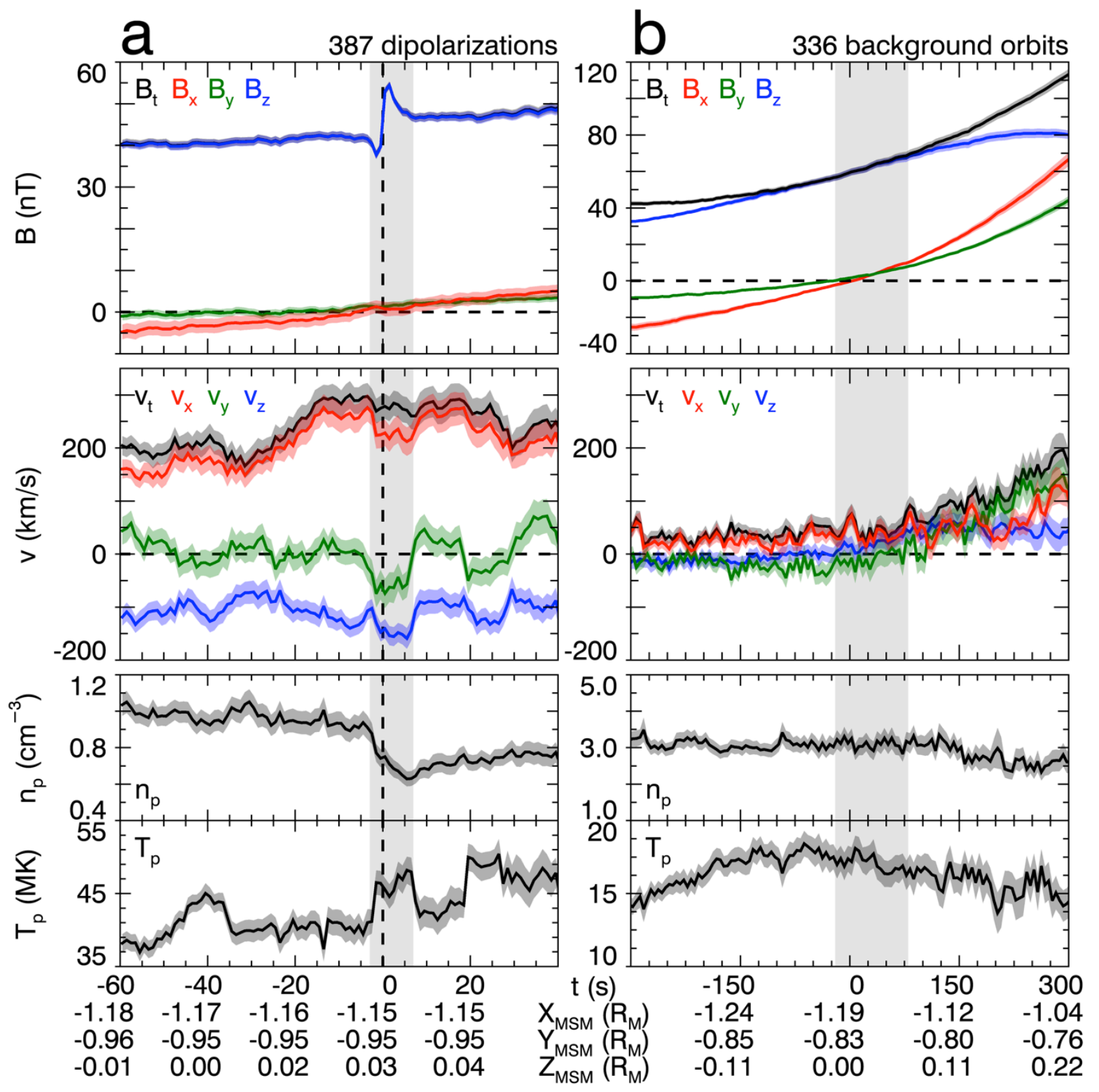

Figure 4. (a) Statistical plasma and magnetic field observations from the 387 dipolarization intervals. (top to bottom) Magnetic field components; plasma flow components; proton density and temperature. Average spacecraft location is listed below the bottom panel. The light grey shaded region spans the statistical dipolarization. (b) Statistical observations from 336

This article is protected by copyright. All rights reserved. 
background orbits, in the same format as (a). The light grey shaded region corresponds to the same range of $Z_{\text {MSM }}$ as in (a). 


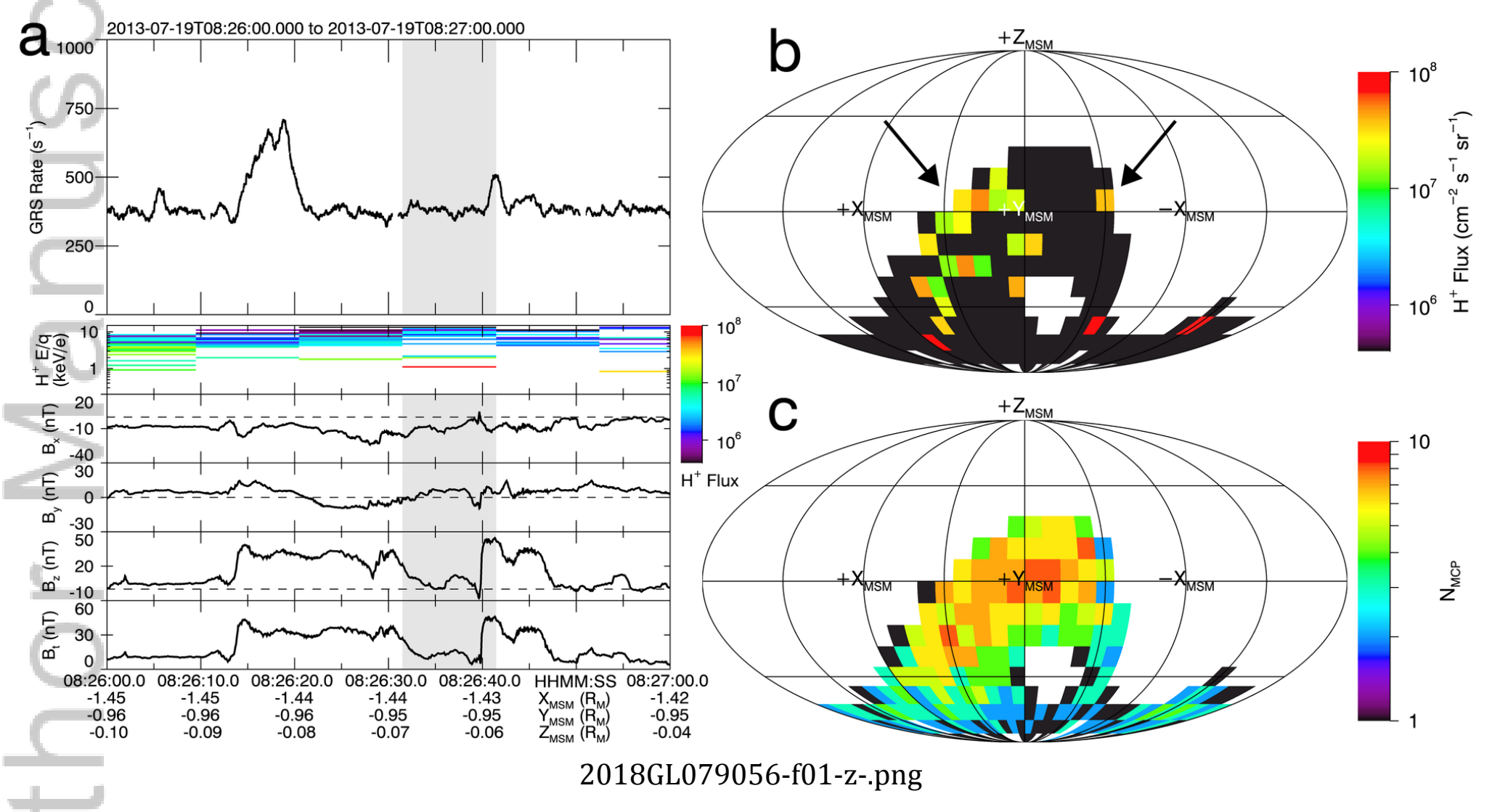

This article is protected by copyright. All rights reserved. 


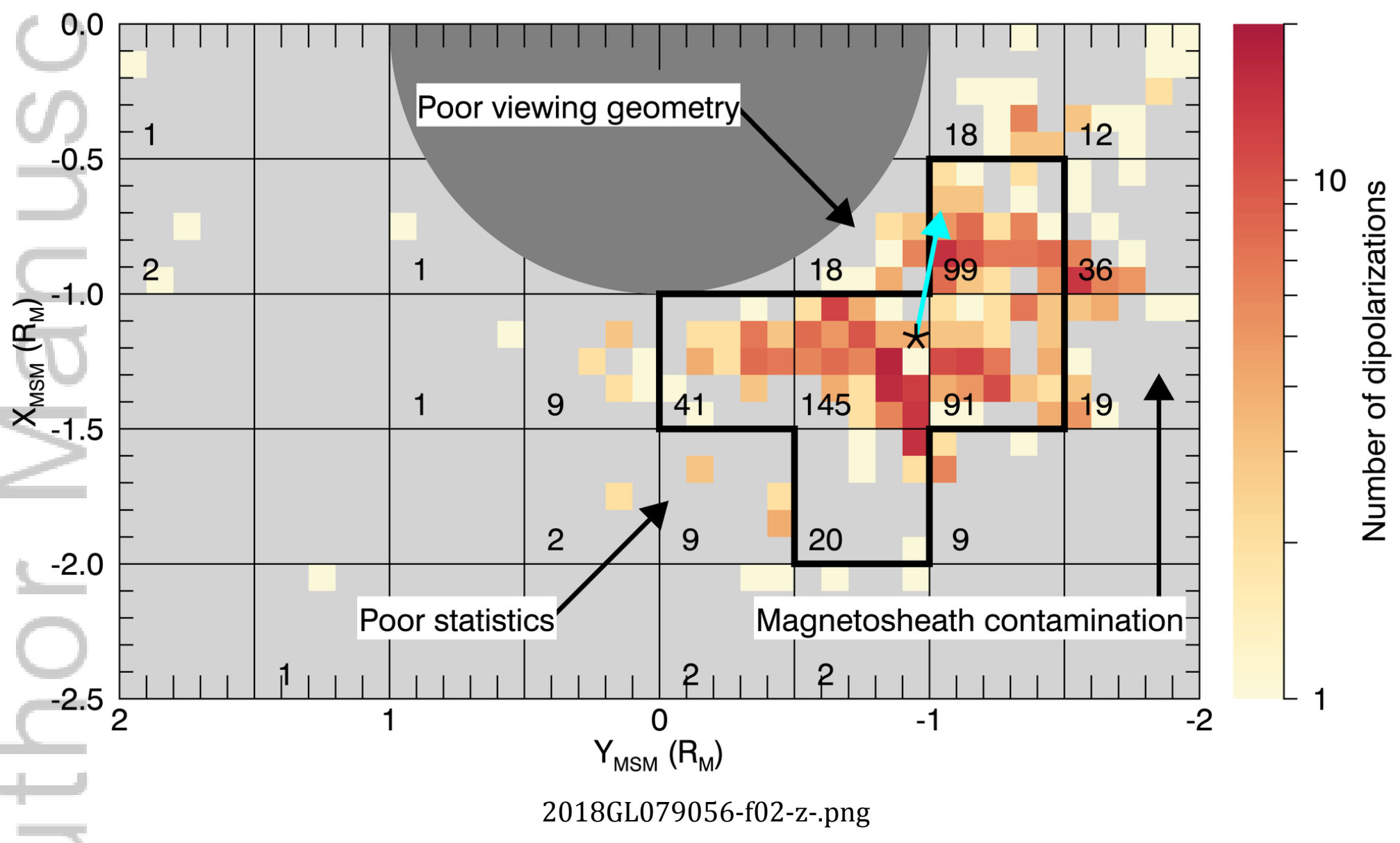

This article is protected by copyright. All rights reserved. 


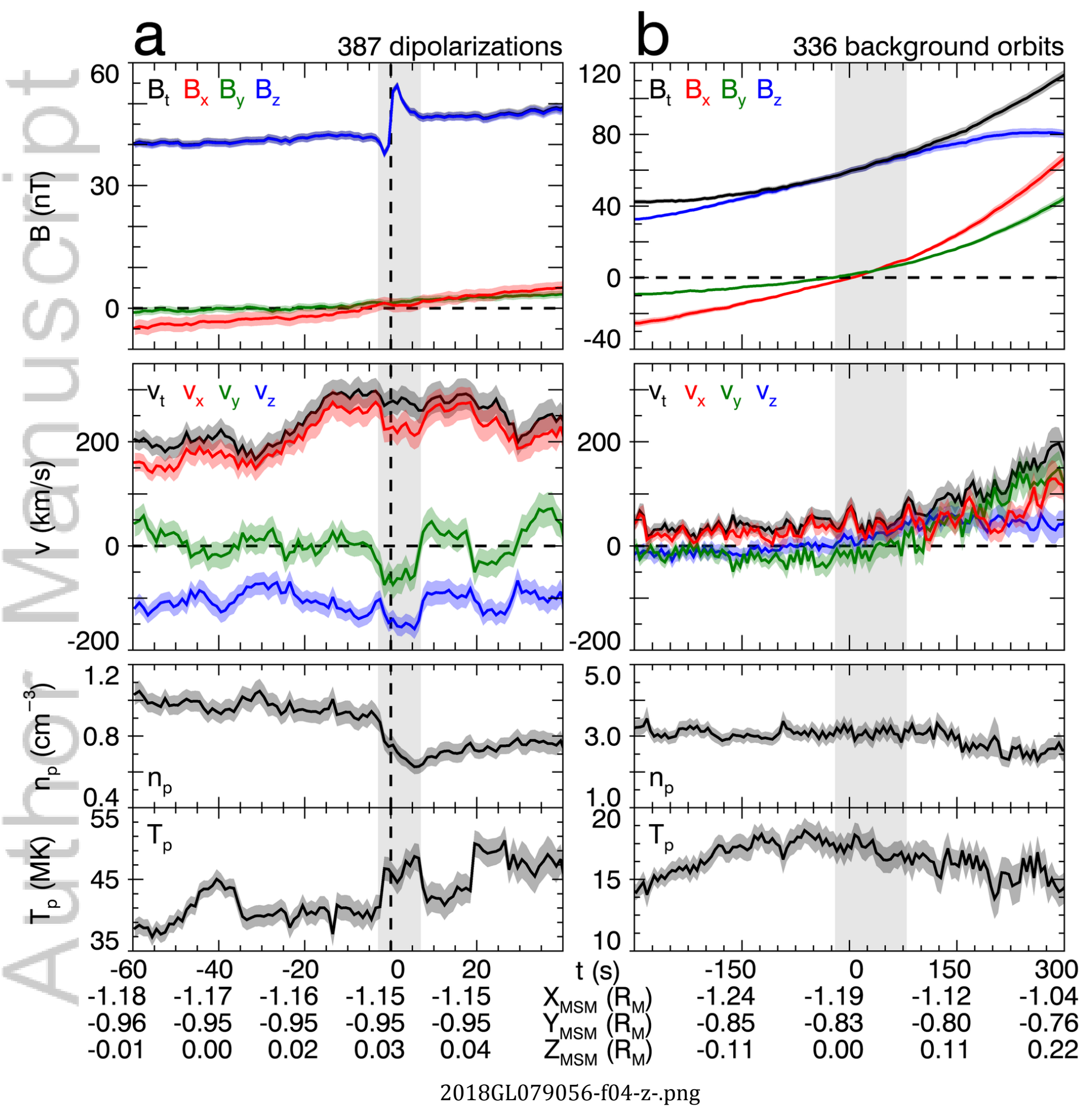

This article is protected by copyright. All rights reserved. 